\title{
Diseño e implementación de una metodología autoformativa para la mejora de la "Comunicación Oral Efectiva” en el ámbito universitario.
}

\author{
Nuria Matarredona-Desantes ${ }^{\mathrm{a}}$, Raúl Oltra-Badenes ${ }^{\mathrm{b}}$, Édgar Pérez-Esteve ${ }^{\mathrm{c}}$, Joan \\ Albert Silvestre-Cerdà ${ }^{\mathrm{d}}$, Maria del Carmen Bas ${ }^{\mathrm{e}}$ y María Jesús Lerma-García ${ }^{\mathrm{f}}$
}

${ }^{a}$ Departamento de Composición Arquitectónica, Universitat Politècnica de València, numade@upvnet.upv.es ; b Departamento de Organización de Empresas, Universitat Politècnica de València, rauloltra@doe.upv.es ; ' Departamento de Tecnología de Alimentos, Universitat Politècnica de València, edpees@upv.es ; ${ }^{d}$ Departamento de Sistemas de Información y Computación, Universitat Politècnica de València, jsilvestre@dsic.upv.es; ${ }^{e}$ Departamento de Matemáticas para la Economía y Empresa, Universitat de València, maria.c.bas@uv.es ; ${ }^{\mathrm{f} D e p a r t a m e n t o ~ d e ~ T e c n o l o g i ́ a ~ d e ~}$ Alimentos, Universitat Politècnica de València, malerga1@tal.upv.es .

\begin{abstract}
The student outcome "Effective Communication" is one of the most studied competence in subjects of different degrees. An inefficient communication can lead to misinterpretations and wrong conclusions. Therefore, it is a very important competence both in the university and in the business field, since the student/graduate must know how to communicate effectively, both orally and in writing, using appropriately the necessary resources and adapting to the characteristics of the situation and the audience. Despite its importance, the disparity in the mastery of the competence between the students makes it difficult to work in the classroom through collective activities. The objective of this study is to design a self-formative methodology that allows the student to detect their weaknesses and strengths in the competence domain and propose teaching-learning formative activities according to their initial level of mastery to improve the acquisition of this transversal competence. The designed methodology has been implemented in a group of students of first course of the Degree in Fundamentals of Architecture obtaining relevant results that indicate an improvement in the domain of the competence.
\end{abstract}

Keywords: Effective oral communication, transversal competence, autonomous learning, self-formative itinerary

\begin{abstract}
Resumen
La competencia transversal "Comunicación Efectiva” es una de las más trabajadas en asignaturas de diferentes materias y ciclos. Una comunicación ineficiente puede llevar a malas interpretaciones y equivocadas conclusiones. Por tanto, se trata de una competencia muy importante tanto en el ámbito universitario como en el ámbito empresarial, ya que el alumno/egresado debe saber comunicarse de manera efectiva, tanto de forma oral como escrita, utilizando adecuadamente los recursos necesarios y adaptándose a las características de la situación y de la audiencia. Pese a su
\end{abstract}


importancia, la disparidad en el dominio de la competencia por parte del alumnado hace difícil su trabajo en el aula a través de actividades colectivas. El objetivo de este estudio es diseñar una metodología autoformativa que permitan al alumno detectar sus debilidades y fortalezas en el dominio de la competencia y proponer actividades formativas de enseñanza-aprendizaje según su nivel inicial de dominio para la mejora de la adquisición de la competencia transversal. La metodología diseñada se ha implantado en un grupo de alumnos de primero en el Grado en Fundamentos de la Arquitectura obteniendo resultados relevantes que indican una mejora en el dominio de la competencia.

Palabras clave: Comunicación Oral Efectiva, competencia transversal, aprendizaje autónomo, itinerario auto-formativo

\section{Introducción}

En los últimos años, las instituciones de educación superior han experimentado diversos cambios condicionados por las exigencias de la sociedad actual. La adaptación de los títulos de grado al Espacio Europeo de Educación Superior (EEES) es uno de los factores ineludibles que han provocado un cambio en la aplicación de las metodologías docentes y una necesidad de adaptar y flexibilizar la oferta formativa a la realidad actual. En particular, la inclusión de las competencias transversales en los planes de estudio es de especial importancia tanto para el ámbito universitario como para el ámbito empresarial. Existe un consenso entre universidad-empresa sobre la necesidad de que los futuros empleados dominen ciertas competencias de cara a su desarrollo profesional. Para el estudiante resulta muy importante adquirir y acreditar su formación en competencias en la universidad y, por otra parte, para el empleador resulta muy relevante conocer el nivel de dominio de las competencias adquirido por los egresados.

Como consecuencia de dicha necesidad la Universitat Politècnica de València (UPV) ha incluido como objetivo estratégico la evaluación de competencias específicas y transversales. La realidad es que se trabajan competencias de ambos tipos, pero debido a la reducida carga docente otorgada a cada asignatura, los profesores suelen priorizar el trabajo de las competencias específicas en detrimento de las transversales. Como resultado de esto, en muchas asignaturas no existe una alineación curricular ya que se evalúan competencias transversales que no se han trabajado en clase o que aun habiéndose trabajado no se han proporcionado actividades formativas para poder mejorar el nivel de dominio de éstas. Es por eso que este estudio se centra en el nivel de dominio de las competencias transversales y en cómo se trabajan en el aula para poder mejorar dicho nivel de dominio competencial.

Para llevar a cabo este estudio ha sido necesario definir el concepto de competencias transversales como "aquellas competencias que son claves y transferibles en relación a una amplia variedad de contextos personales, sociales, académicos y laborales a lo largo de la vida. En este sentido, constituyen una parte fundamental del perfil profesional y del perfil formativo de todas o de la mayoría de las titulaciones. Son competencias que incluyen un conjunto de habilidades cognitivas y meta-cognitivas, conocimientos instrumentales y 
actitudinales de gran valor para la sociedad del conocimiento" (ICE, 2017). Se trata, por tanto, de un saber hacer muy complejo, por lo que es necesario concretarlas en resultados de aprendizaje más específicos. Por ello, será necesario recurrir a estrategias metodológicas que propicien el cambio de roles. Ya no se puede utilizar una metodología 'tradicional' basada en clases magistrales y problemas que el profesor resuelve en el aula. Incluso las prácticas de laboratorio deben cambiar su enfoque para conseguir nuevos objetivos y lograr que los alumnos adquieran una serie de capacidades que, por lo general, antes no se tenían en cuenta ni se evaluaban, como son la capacidad de trabajo en grupo, la capacidad de realizar exposiciones orales, etc. (Oltra-Badenes y Gil-Gómez 2015). Para alcanzar las competencias es necesario realizar acciones formativas. Dichas acciones deben estar basadas en metodologías activas para la formación de competencias y en métodos de participación del alumno que generan un aprendizaje más profundo, significativo y duradero (Fernández, 2006).

El presente estudio de innovación docente pretende trabajar la competencia transversal "Comunicación Oral Efectiva" que parece ser una de las competencias en las que existe un mayor consenso respecto a la importancia que tiene para los futuros egresados. Según el proyecto institucional desarrollado por la UPV, comunicarse efectivamente significa tener desarrollada la capacidad de transmitir conocimientos y expresar ideas y argumentos de manera clara, rigurosa y convincente, tanto de forma oral como escrita, utilizando los recursos apropiados adecuadamente y adaptándose a las circunstancias y al tipo de público. Es importante diferenciar dos dimensiones dentro de esta competencia: la comunicación oral y la escrita (Universitat Politècnica de València, 2012).

El dominio de la competencia de comunicación efectiva implica la eficacia en la comunicación de ideas, conocimientos y sentimientos a través de la palabra y el escrito tanto en situaciones conversacionales/escritas y en actividades grupales como en presentaciones públicas ante audiencias más o menos numerosas (Villa y Poblete, 2007). Los estudiantes que acceden a la universidad ya han adquirido cierto bagaje instrumental de técnicas y estrategias para una buena comunicación, pero es importante que en la universidad se continúe trabajando dicha competencia teniendo en cuenta sus importantes implicaciones académicas, profesionales y personales. Desde un punto de vista estrictamente académico, las interacciones verbales y escritas constituyen la clave del proceso de enseñanza-aprendizaje, ya que facilitan la actividad colaborativa, posibilitan la interiorización del conocimiento y son fundamentales para conseguir un buen rendimiento académico. En la vida profesional resulta imprescindible saber transmitir ideas, conocimientos y sentimientos de forma precisa para obtener una mayor eficacia. Por último, desde la perspectiva personal, el hecho de poder hablar en público con seguridad y sin nerviosismo, así como la facilidad de expresarse por escrito sin dificultades comporta un incremento de la seguridad personal y refuerza la autoestima (Sayós, R., 2013).

En general, pensamos que dominamos dicha competencia debido a la necesidad que tenemos de comunicar información entre nosotros. Sin embargo, en muchas situaciones existe una gran diferencia entre lo que pretendemos decir y el mensaje que le llega al receptor. Es por ello que los alumnos se enfrentan al desarrollo de esta competencia con 
niveles de dominio muy dispares debido a las diferentes capacidades innatas y a los diferentes ritmos de adquisición de esta competencia. Además, esta disparidad se puede acentuar si los alumnos están matriculados en titulaciones y en ciclos diferentes. Por tanto, surgen distintas problemáticas en cuanto a la adquisición de esta competencia transversal:

-¿Qué nivel de dominio inicial de esta competencia transversal tienen los alumnos de distintas titulaciones y ciclos?

-¿Cómo se trabaja dicha competencia en clase?

-¿Qué herramientas de evaluación/diagnóstico se usan para analizar el nivel de dominio de la competencia?

-¿Existen herramientas de apoyo y mejora que guíen al alumno a la adquisición/mejora de la competencia?

-¿Qué actividades formativas se deben proponer para mejorar el dominio de la competencia?

En este estudio hemos abordado algunas de las problemáticas presentadas anteriormente mediante la elaboración de una metodología autoformativa que ayude a los estudiantes universitarios a la mejora de la adquisición de la competencia transversal. A continuación, se plantea el objetivo de este estudio, el diseño de la metodología con acciones que abordan la problemática anterior y la implementación en un estudio piloto.

\section{Objetivo}

El objetivo fundamental del presente trabajo es presentar la metodología diseñada en el marco del Proyecto de Mejora e Innovación Docente PIMECOE para fomentar el desarrollo mediante aprendizaje autónomo de la dimensión oral de la competencia transversal CT08 «Comunicación Efectiva» en el ámbito universitario, así como compartir los resultados obtenidos a partir de su implementación experimental en el primer curso de Grado en Fundamentos de la Arquitectura cuyo análisis permitirá generar una ulterior versión revisada de las herramientas asociadas a esta metodología.

\section{Diseño de la metodología PIMECOE}

La metodología autoformativa diseñada para la mejora del dominio del nivel inicial de la CT "Comunicación Oral Efectiva” se ha basado en 4 fases fundamentales. La figura 1 muestra cada una de las fases de la metodología y el orden de ejecución de estas. 


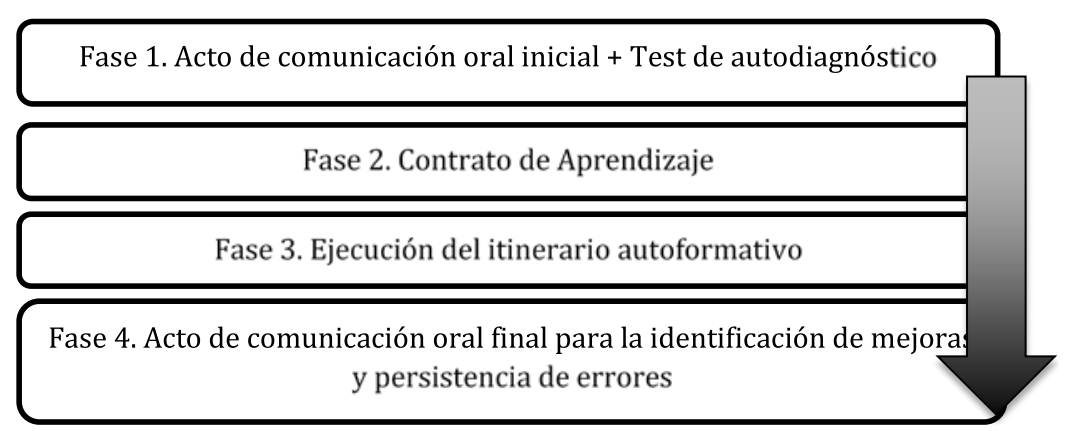

Fig. 1 Fases de la metodología autoformativa de la competencia "Comunicación Oral Efectiva"

A continuación se describe el objetivo y las acciones de cada una de las fases de la metodología.

\section{Fase 1: Acto de comunicación final + Test de autodiagnóstico}

La primera fase de la metodología corresponde a la detección del nivel inicial del dominio de la competencia que presentan los alumnos. Para ello, el profesor propone un acto de comunicación oral inicial que permite testear su nivel inicial y evaluar mediante la rúbrica de la evaluación diseñada por la UPV los indicadores de dominio de la competencia. El feedback que reciben los alumnos permite determinar las fortalezas y los puntos débiles del alumnado en relación con los indicadores básicos y avanzados que definen cada nivel de dominio de la CT.

Seguidamente, el estudiante debe realizar el test de autodiagnóstico diseñado en el marco del proyecto PIMECOE utilizando la experiencia personal del acto comunicativo inicial. La finalidad principal del test de autodiagnóstico de la Competencia transversal Comunicación Oral Efectiva no es otra que la de orientar y ayudar al alumno en la mejora de sus habilidades para conseguir una comunicación oral efectiva a partir de una serie de indicadores mensurables facilitados por el propio test. De este modo, el test persigue un doble propósito: por un lado, busca ser una herramienta que ayude al estudiante a medir y evaluar de forma general sus habilidades en la comunicación oral efectiva, y por otro, es un instrumento útil de cara a la mejora de las mismas.

Dicho autodiagnóstico será clave para que el estudiante pueda identificar aquellos indicadores que debe mejorar. Con este fin, el test se ha dividido en seis bloques atendiendo a la rúbrica de evaluación de la competencia transversal Comunicación Oral Efectiva elaborada por la UPV. A su vez, cada una de estos bloques contiene una serie de indicadores cuyo valor vendrá dado entre 1 (nunca o nada) y 5 (siempre o mucho). Atendiendo a la puntuación final obtenida en cada indicador y/o bloque, y dependiendo del nivel de dominio del estudiante (nivel I - $1^{\circ} 02^{\circ}$ de grado; nivel II - $3^{\circ}$ o $4^{\circ}$ grado; nivel III Máster), al final del test de autodiagnóstico el estudiante encontrará un conjunto de actividades a realizar para mejorar cada uno de los indicadores y/o bloques y poder conseguir así un nivel óptimo de la competencia transversal Comunicación Oral Efectiva. 
El conjunto de actividades forma el itinerario autoformativo que se destallará en su fase correspondiente.

El test de autodiagnóstico se puede encontrar en la página web del proyecto PIMECOE (Silvestre-Cerdá et al, 2017).

\section{Fase 2: Contrato de aprendizaje}

El contrato de aprendizaje corresponde a la segunda fase de la metodología. Se trata de un documento que permite ayudar y guiar al estudiante en su proceso de aprendizaje de la competencia. Según Lobato (2006) el contrato de aprendizaje se define como "un acuerdo establecido entre el profesor y el estudiante para la consecución de unos aprendizajes a través de una protesta de trabajo autónomo, con una supervisión por parte del profesor y durante un período determinado. En un contrato de aprendizaje es básico un acuerdo formalizado, una relación de contraprestación recíproca, una implicación personal y un marco temporal de ejecución”.

Por tanto, este documento nace de un acuerdo y de una negociación entre el estudiante y el profesor, ya que pactan qué, cómo y cuándo van a trabajar las actividades recogidas en el documento "itinerario autoformativo" con el objetivo de mejorar su dominio de la competencia comunicación efectiva. Es un método de trabajo autónomo puesto que la responsabilidad el proceso de aprendizaje recae sobre el estudiante. De esta forma se trabaja también el aprendizaje autónomo de los estudiantes ya que éste asume una parte importante de la responsabilidad de la organización de su trabajo adaptándolo a su propio ritmo (Ruiz, 2006).

\section{Fase 3: Ejecución del itinerario autoformativo}

La tercera fase corresponde a la ejecución del itinerario autoformativo, consistente en un documento de trabajo en el que se especifica, en función de las respuestas proporcionadas por el alumnado en el test de autodiagnóstico, el conjunto de actividades que el alumno deberá trabajar para mejorar su nivel de domino de la competencia. De forma autónoma y cumpliendo los plazos establecidos en el contrato de aprendizaje, el alumno trabajará actividades recomendadas para fortalecer los puntos débiles detectados en la guía de autodiagnóstico en la competencia de comunicación oral. El documento se encuentra también implementado on-line en la web del proyecto PIMECOE.

\section{Fase 4. Acto de comunicación oral final para la identificación de mejoras y permanencia de errores.}

En la última fase de la metodología autoformativa propuesta, se plantea la realización de un acto de comunicación oral, pudiendo ser éste el mismo o similar al inicial. El objetivo de este acto final es evaluar de nuevo al alumnado mediante los indicadores de la rúbrica de la universidad e identificar las mejoras que ha tenido en la comunicación oral y los errores o puntos débiles persistentes que no ha logrado mejorar. De esta manera, el alumno dispone de un feedback de sus resultados que le permiten analizar su evolución en cuanto a la adquisición de la competencia y detectar la permanencia de errores que continúa teniendo 
en el desarrollo de la habilidad. Además, esta última fase permite analizar si ha habido diferencias entre el pre y post análisis.

\section{Implementación experimental de la metodología PIMECOE}

\subsection{El porqué de una experiencia piloto}

El testeo de la metodología autoformativa se contempla como una fase previa fundamental en el proceso de diseño de la metodología PIMECOE. El ensayo de las herramientas en su versión preliminar permite recabar datos de carácter tanto cuantitativo como cualitativo que resultan imprescindibles para incrementar su garantía de éxito entre el alumnado universitario.

\subsection{Contexto académico}

La metodología PIMECOE se implementa de manera experimental en la asignatura Introducción a la Arquitectura (IAR) del primer curso del Grado en Fundamentos de la Arquitectura. A pesar de no ser punto de control de esta competencia transversal, la CT08 se trabaja habitualmente en el desarrollo del curso.

IARexp, acrónimo de esta experiencia piloto, se desarrolló durante el periodo lectivo de dicha asignatura que se corresponde con el primer semestre del curso 2017/2018. El objetivo de esta asignatura es el de reflexionar acerca del concepto de arquitectura y el papel que el ejercicio profesional de esta disciplina debe jugar en nuestra sociedad, así como aproximarse a los conceptos teóricos base del proyecto arquitectónico desde el análisis de nuestro entorno.

Son dos los grupos objetivo que participan en esta experiencia. El grupo C está compuesto por 45 estudiantes y su lengua vehicular es el valenciano. El grupo D, formado por 60 estudiantes se comunica en castellano.

Cabe destacar que, paralelamente al transcurso de esta asignatura, el estudiante comienza a familiarizarse con el mundo universitario y todas aquellas herramientas que le son propias lo que supone un contexto muy específico de desarrollo del alumnado.

\subsection{Cronograma}

La aprobación del proyecto "Autodiagnóstico del nivel de dominio inicial de la competencia transversal "Comunicación Efectiva” y su mejora a través de un itinerario autoformativo" (PIME/2017/B/025-14) se produce en septiembre de 2017, coincidiendo con el inicio del curso 2017/2018. Puesto que la duración establecida del proyecto PIMECOE es de un año, el cronograma del proyecto preveía el desarrollo de la herramienta durante el primer semestre de curso y su puesta en marcha durante el segundo semestre. Sin embargo, la oportunidad de realizar una experiencia piloto durante el primer semestre, a pesar del esfuerzo que supone la condensación del cronograma de proyecto, se considera una valiosa aportación para el proyecto puesto que permite que la versión a implementar durante el segundo semestre cuente ya con cierta retroalimentación por parte del estudiantado. 
El cronograma de actividades para el alumnado de IARexp vinculadas con el PIMECOE se integra absolutamente dentro de la planificación de la asignatura y refuerza el desarrollo de las competencias específicas y transversales a desarrollar.

\subsubsection{Desarrollo de la tarea "Opiniones sobre arquitectura"}

La primera tarea en la que la metodología PIMECOE se incorpora a la asignatura corresponde a la tercera tarea desarrollada durante el curso: “Opiniones sobre arquitectura”. Esta corresponde a la segunda unidad del curso dedicada precisamente a reflexionar acerca del concepto de arquitectura. Tras el debate colectivo en el aula, se invita al alumnado a conocer la idea de arquitectura que manifiestan arquitectos de prestigio de modo que puedan aproximarse al concepto desde otra perspectiva.

Para ello, cada estudiante debe localizar una definición de arquitectura que le resulte sugerente. Una vez seleccionada, se comienza a profundizar en la figura del arquitecto autor de la misma. De entre su obra construida se escoge un caso de estudio sobre el que realizar un incipiente análisis arquitectónico. Finalmente, se pretende reflexionar sobre la relación entre la definición de arquitectura y la obra realizada.

Los resultados de aprendizaje esperados evidentemente contemplan ampliar su concepto de arquitectura, pero también el familiarizarse con figuras relevantes del panorama mundial que hasta el momento desconocían. Este tipo de actividades persiguen el desarrollo de competencias transversales como el desarrollo del pensamiento crítico y la comunicación efectiva.

\subsubsection{Exposición oral "Opiniones sobre arquitectura"}

Al tratarse de un estudio individual, resulta crucial que los resultados sean compartidos entre los compañeros para lograr los resultados de aprendizaje esperados. Para ello, se diseñan unas sesiones de exposición oral a modo de foro. Estas exposiciones orales corresponden a la fase 1 del proyecto PIMECOE. Al término de las mismas, se conocerán tantas obras y autores como alumnos hayan expuesto. De esta manera, se ponen sobre la mesa diversas ideas sobre arquitectura al mismo tiempo que su iniciática cultura arquitectónica comienza a cultivarse.

Considerando el elevado número de estudiantes en cada uno de los grupos, se adopta el sistema de presentación PechaKucha para las mismas. Este dinámico formato surge en Japón en 2003 de mano del estudio de arquitectura Kein Dytham Architecture (PechaKucha:2018), y consiste en acompañar el discurso oral con 20 imágenes. Cada una de ellas permanece visible durante 20 segundos que, una vez transcurridos, dejan paso a la siguiente, siendo imposible modificar dicha transición automática. En este caso, el formato se adapta de un 20x20 a un 10X20, de manera que cada una de las exposiciones tiene una duración final de 3 minutos y 20 segundos. 


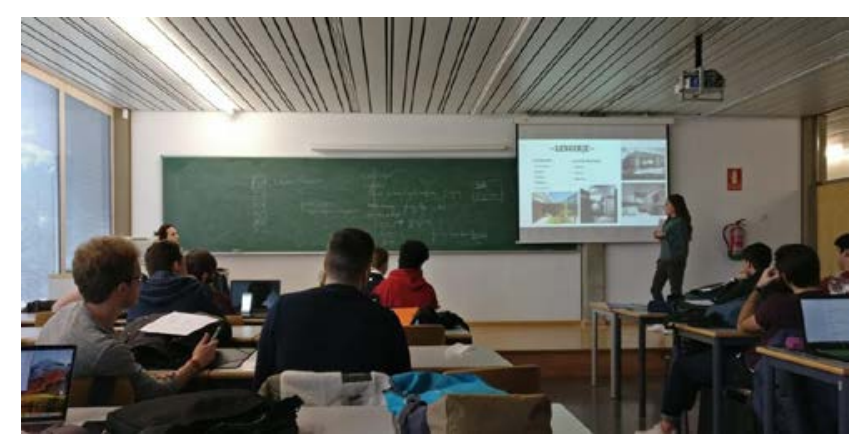

Fig 2. Estudiante presentado su tarea.

En aras de fomentar el rol activo de los alumnos escuchantes, y considerando los beneficios de la evaluación por pares, se les invita a ejercer la función de revisores. Para ello, se les proporciona una rúbrica a modo de guía con la que evaluar tanto el contenido del trabajo presentado como el desarrollo de la exposición oral. Se usa la rúbrica de evaluación diseñada por la UPV en la que se incluyen cinco indicadores y su nivel de dominio, siendo (A: excelente, ejemplar; B: adecuado, bueno; C: en desarrollo, D: no alcanzado). Los indicadores analizados son:

I1: Transmite información relevante y sabe responder las preguntas que se le formulan

I2: Las exposiciones están estructuradas de modo coherente

I3: Utiliza el lenguaje con corrección gramatical: semántica, sintáctica y ortográfica

I4: Su comunicación no verbal transmite tranquilidad

I5: Utiliza medios de apoyo de modo pertinente

Además, se incluyen dos casillas para que se valore cualitativamente la exposición y el contenido del trabajo. Junto a la rúbrica, un listado de verificación con tantas casillas como estudiantes realizan la exposición oral.

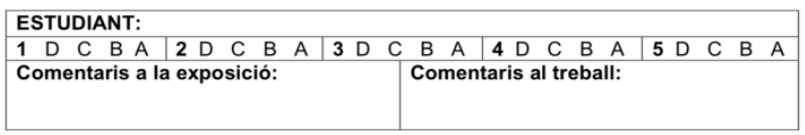

Fig 3. Listado de verificación.

Para el adecuado procesado de los datos, cada uno de los estudiantes cuenta con un código asignado que complementa su identificación con nombre y apellidos. La participación en estas exposiciones, así como el adecuado desarrollo de su función como revisores se fomenta con un incremento en su calificación final. Por otro lado, el profesorado evalúa los mismos ítems que el estudiantado. Para poder dedicar mayor atención a los contenidos, miembros del PIMECOE se dedican específicamente a la evaluación de la competencia de comunicación efectiva.

\subsubsection{Presentación de la metodología autoformativa del proyecto PIMECOE}

Una vez finalizadas las sesiones de exposición oral, se invita a los estudiantes a reflexionar acerca de la importancia de la comunicación oral efectiva y en la repercusión de una buena

(c)) EY-NC-ND 2018, Universitat Politècnica de València

Congreso In-Red (2018) 
exposición en la comprensión del trabajo desarrollado. Evidentemente, el estudiantado se muestra interesado en mejorar esta competencia.

En este momento, se presenta la metodología autoformativa del proyecto PIMECOE y se ponen a su disposición las herramientas diseñadas hasta el momento: la página web PIMECOE, el “Test de autodiagnóstico", el "Itinerario autoformativo” y el "Contrato de aprendizaje”.

\subsubsection{Diagnóstico del estado de dominio inicial de la competencia}

El diagnóstico del estado de dominio inicial de esta competencia se obtiene mediante dos vías. La primera de ellas, a través de la realización del propio "test de autodiagnóstico" ofrecido por PIMECOE, mediante el que pueden detectar su principales carencias y virtudes. Por otro lado, cada estudiante recibe mediante un informe elaborado por el equipo de PIMECOE la retroalimentación de sus compañeros que han actuado como evaluadores.

El resultado de este diagnóstico permite que cada estudiante tome conciencia de las áreas en las que debe trabajar para obtener mejores resultados en sus exposiciones.

\subsubsection{Firma del contrato de aprendizaje y realización del itinerario autoformativo}

Una vez realizada la fase 1 de la metodología correspondiente a la actividad oral inicial y al test de autodiagnóstico, el alumnado se compromete a realizar las actividades del itinerario autoformativo basándose en la puntuación del test y según su compromiso en cantidad y tiempo reflejado en el contrato de aprendizaje firmado entre estudiante-profesor. Estas actividades corresponden a las fases 2 y 3 de la metodología propuesta.

\subsubsection{Desarrollo de la tarea "La casa"}

Finalmente, los estudiantes tienen la posibilidad de comprobar su propia evolución y la de sus compañeros durante el desarrollo de la sexta práctica: “La casa”. Esta tarea corresponde a la fase final del curso y presenta un carácter concluyente, puesto que requiere de la aplicación de los contenidos trabajados a lo largo del curso a un caso de estudio. En este caso, cada estudiante selecciona una vivienda unifamiliar de entre un listado ofertado por el profesorado.

En este caso, la presente tarea se evalúa en primera instancia por los propios compañeros, empleando la herramienta de revisión por pares que ofrece la propia plataforma educativa de la UPV, PoliformaT (Matarredona-Desantes, 2017). Posteriormente, y con la misma intención perseguida en la práctica anteriormente expuesta, los trabajos son presentados oralmente en unas sesiones dedicadas expresamente a ello.

De esta manera, el alumnado termina aproximándose a un importante número de casos de estudio en tres niveles de profundidad diferente (nivel muy alto, el analizado personalmente; nivel alto, los dos casos que ha revisado a través de la plataforma y finalmente, nivel medio, aquellos presentados en el aula).

\subsubsection{Exposición oral "La casa”}

Durante las dos últimas sesiones en aula, los estudiantes presentan oralmente la tarea. En este momento se pone en evidencia el trabajo realizado autónomamente en relación con el desarrollo de la competencia transversal de comunicación efectiva y la deseable evolución en el grado de dominio de la misma. 
La evaluación se realiza siguiendo el mismo sistema descrito en la tarea anterior, tanto por pares como por el profesorado y los miembros del PIMECOE. Del mismo modo, la participación del alumnado en estas exposiciones, así como su función como revisores se fomenta con un incremento en su calificación final.

\section{Resultados}

A continuación, se muestran los resultados obtenidos de la implantación de la metodología autoformativa para la mejora del dominio de la CT Comunicación Oral Efectiva, propuesta en el proyecto PIMECOE, en la experiencia piloto en el Grado de Arquitectura. En primer lugar, se presentan los resultados cuantitativos y posteriormente se realizar un estudio cualitativo de la experiencia.

\subsection{Evaluación cuantitativa}

Los resultados cuantitativos de este estudio se basan en los resultados de la rúbrica obtenidos en la evaluación los actos de comunicación oral al inicio y al final de la metodología aplicada. De esta forma, los resultados permiten realizar una comparativa entre el antes y el después de la aplicación de la metodología propuesta (pre-test y post-test) y observar mejoras en el desarrollo de la competencia trabajada.

Para ello se cuenta con una muestra de 46 alumnos en el pre-test y 36 alumnos en el posttest de los cuales 30 han realizado ambas fases. En la figura 4 se muestran los resultados del porcentaje de los distintos niveles de dominio obtenidos para todos los indicadores evaluados en la rúbrica en el pre-test (46 alumnos) y post test (36 alumnos). Los resultados muestran que el porcentaje de "A: nivel excelente" y "B: nivel adecuado" ha aumentado en el post-test, mientras que los niveles "C: en desarrollo" y " $\mathrm{D}$ : no alcanzado" han disminuido después de implementar la metodología autoformativa.

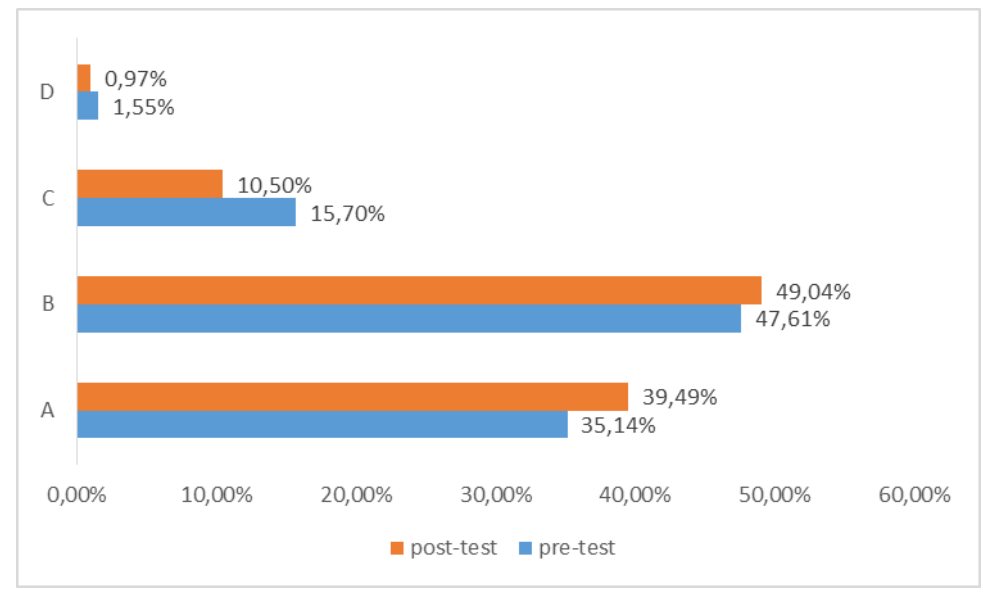

Fig. $4 \%$ Dominio en el pre-test y post-test

La figura 5 muestra los resultados del mismo análisis pero desagregado por indicadores evaluados. En general se observa que el porcentaje de "A" aumenta para todos los 
indicadores en el post-test, es decir que los alumnos obtienen una puntuación de dominio excelente en todos los indicadores una vez realizada la metodología autoformativa propuesta. De forma contraria, el porcentaje de "C" y "D" disminuye en el post-test, concluyendo, por tanto, que hay menos deficiencias en el dominio de la competencia. En cuanto al nivel de dominio "B: adecuado" de los indicadores, se mantiene aproximadamente en todos, exceptuando el "I4: Su comunicación no verbal transmite tranquilidad" en el que se observa una mejora en el post-test. Este resultado puede ser debido a la tranquilidad de los alumnos al exponerse a una segunda comunicación oral. Este resultado permite concluir que cuantas más exposiciones realiza el alumno y más trabaja la competencia, mayor tranquilidad transmite en las exposiciones orales.

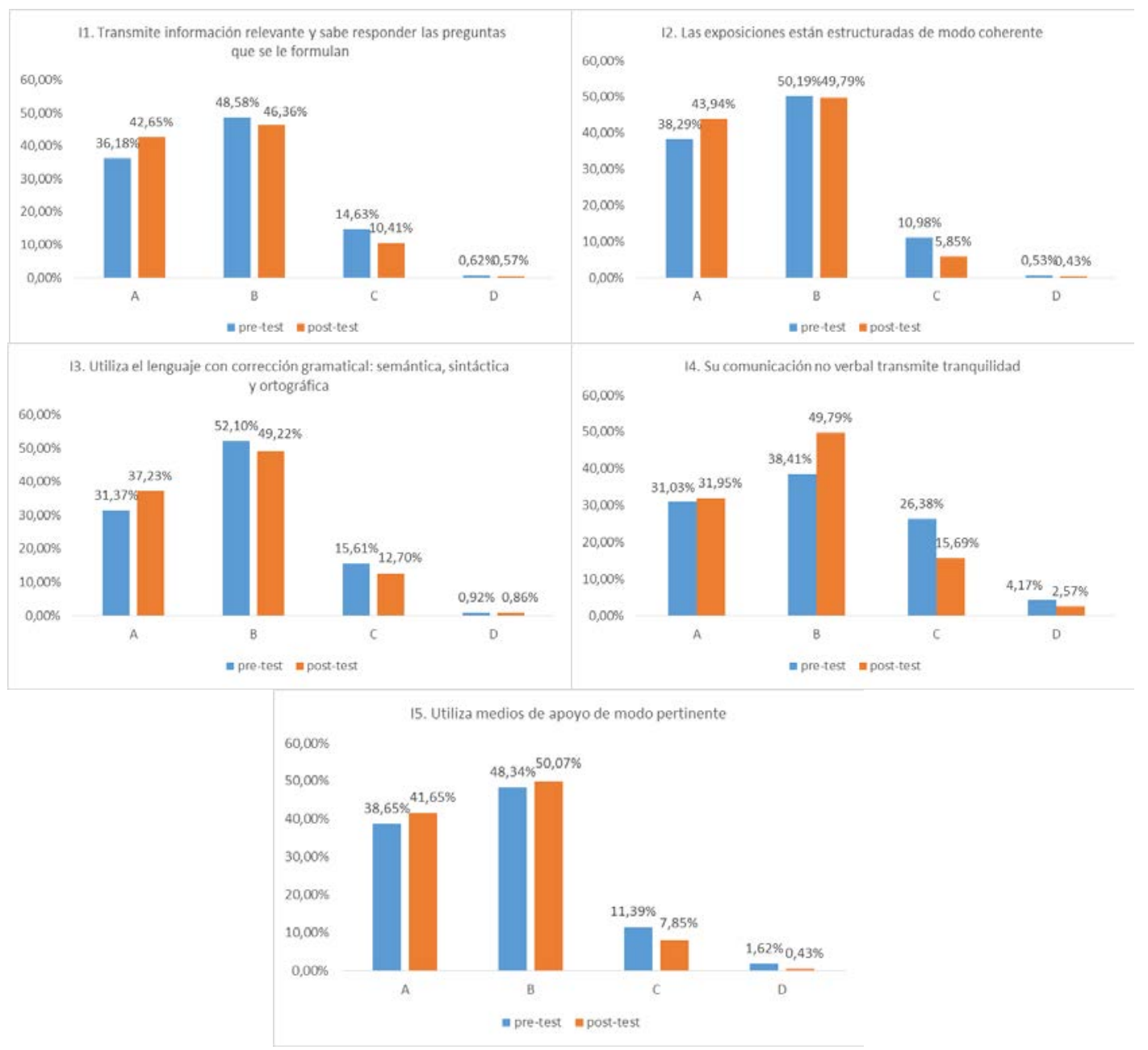

Fig 5. \%Dominio en cada Indicador en el pre-test y post-test

Finalmente se ha realizado un estudio con datos pareados centrado en los 30 alumnos que hicieron el acto comunicativo en la fase 1 y en la fase 4 de la metodología, pudiendo 
realizar una comparativa de su evolución. Para ello, se ha calculado para cada alumno el porcentaje total de cada una de las categorías de dominio (para todos los indicadores) en el pre-test y post-test y se ha contabilizado el número de alumnos en los que dicho porcentaje ha sido mayor en el post-test que en el pre-test. La tabla 1 muestra los resultados obtenidos.

Tabla 1. Resultados del estudio de evolución

\begin{tabular}{ccc}
\hline $\begin{array}{c}\text { Dominio de la } \\
\text { competencia }\end{array}$ & Nombre & \# Post $>$ Pre \\
\hline A & Excelente, ejemplar & 18 \\
B & Adecuado, bueno & 14 \\
C & En desarrollo & 11 \\
D & No alcanzado & 5 \\
\hline
\end{tabular}

Los resultados indican que 18 de los 30 alumnos han obtenido un mayor porcentaje del nivel de dominio $\mathrm{A}$ en el post-test que en el pre-test, para todos los indicadores de la rúbrica. En cuanto al dominio B, aproximadamente la mitad de alumnos han obtenido mejor porcentaje de nivel B en el pre-test y la otra mitad en el post-test. Sin embargo, sí que se observa un menor número de alumnos, 11 y 5 , en los niveles $\mathrm{C}$ y D respectivamente, que obtienen mayor porcentaje de estos dominios en el post-test y este resultado es positivo teniendo en cuenta que cuanto menor sea el porcentaje de los niveles C y D mejor para el alumno.

Estos resultados son indicativos de una evidente mejora de los estudiantes en el dominio de la competencia transversal "Comunicación Oral Efectiva" mediante el uso de la metodología autoformativa propuesta en este estudio.

\subsection{Evaluación cualitativa}

Los resultados cualitativos de la presente experiencia, IARexp, se basan en la información recogida mediante tres encuestas realizada al alumnado de la asignatura.

La primera de ellas, al término del mismo test de autodiágnostico, está formada por una sencilla pregunta abierta en la que se pregunta la opinión del alumnado acerca de la herramienta. Los estudiantes destacan como positivo el proceso de reflexión que se detona con su realización así como el hecho de que las propias preguntas del test ya desvelan ítems relacionados con la comunicación oral efectiva que desconocían. En general, las cuestiones del test de autodiagnóstico se han percibido sencillas de comprender, pero complejas de responder. Manifiestan cierta dificultad para discernir cual es el "grado de cumplimiento".

La segunda encuesta en la que el alumnado de IARexp participa no tiene como objeto principal la aplicación de la metodología PIMECOE sino que se refiere a la evaluación final de la asignatura. La valoración del PIMECOE aparece implícita en los ítems "Práctica 3", 
“Práctica 6” y "Exposiciones orales”, en los que se introduce una pregunta con respuesta abierta. En general, se manifiesta en diversas ocasiones el interés por las sesiones de exposición oral como espacios de aprendizaje que deben fomentarse. No obstante, por el contrario, al menos tres estudiantes muestran su preocupación por la comunicación oral y califican las exposiciones orales de "mal trago".

Finalmente, la tercera encuesta, sí ha sido diseñada explícitamente para conocer el grado de satisfacción general del estudiantado con la metodología PIMECOE, así como la relación entre el tiempo invertido y los resultados obtenidos. Desafortunadamente, no se han obtenido datos suficientes a través de esta vía puesto que el curso de la experiencia piloto ya había finalizado cuando se diseñó la encuesta. Hay que tener en cuenta que la primera implantación de la metodología propuesta es una experiencia piloto que se ha llevado de forma paralela al diseño de las diferentes fases de la metodología, por lo que no se tienen datos de esta última encuesta.

\section{Conclusiones}

El desarrollo de la competencia transversal "Comunicación Efectiva” y, en particular, su dimensión oral que es la que se ha trabajo en este estudio, es muy importante dentro del ámbito universitario, ya que el alumno debe expresar sus dudas, sus opiniones e incluso realizar exposiciones orales a lo largo de su carrera académica. Además, dicha competencia es muy valorada y requerida en el ámbito empresarial por lo que es muy importante que se adquiera y trabaje en la universidad. Sin embargo, debido al reducido tiempo que tienen los profesores para trabajar las competencias transversales en clase, no se suele profundizar en su aprendizaje. La metodología autoformativa propuesta en este estudio permite trabajar al alumno para mejorar la adquisición de la competencia, midiendo el nivel inicial de dominio y ofreciéndole un itinerario autoformativo para su mejora. Esta metodología no requiere de mucho tiempo de clase, sino que se trata de un aprendizaje autónomo por parte del alumno pero a la vez tutorizado por el profesor para cumplir con un contrato de aprendizaje establecido por ambas partes. Los resultados cuantitativos de la implementación de la metodología autoformativa en una experiencia piloto formada por alumnos de primer grado evidencia mejoras en la adquisición de la competencia. Además, el estudio cualitativo muestra un alto grado de interés por parte de los estudiantes de que se fomenten actividades de aprendizaje que ayuden a mejorar el dominio de la competencia. Por otra parte, este estudio cualitativo también nos ha servido para realizar una revisión en profundidad del test de autodiagnóstico y mejorar el enunciado y respuesta de cada una de las preguntas planteadas. El objetivo a corto plazo es implantar la metodología propuesta en dos másteres de la universidad y volver a analizar los resultados y las encuestas para mejorar de nuevo la metodología. Creemos que se trata de una metodología muy útil, aplicable a cualquier grado y máster de la universidad y extensible al desarrollo de la misma para el dominio de otras competencias transversales. 


\section{Agradecimientos}

El presente trabajo ha sido desarrollado en el marco del Proyecto de Innovación y Mejora Docente "Autodiagnóstico del nivel de dominio inicial de la competencia transversal "Comunicación Efectiva” y su mejora a través de un itinerario autoformativo" (Ref.: PIME/2017/B/025-14) financiado por el Vicerrectorado de Estudios, Calidad y Acreditación de la Universitat Politècnica de València a quien los autores agradecen expresamente este apoyo.

Asimismo, el equipo PIMECOE agradece a los estudiantes de la asignatura Introducción a la Arquitectura su participación en esta experiencia. Su contribución ha resultado fundamental para desarrollar una metodología con mayores garantías de éxito entre el alumnado.

\section{Referencias}

FERNÁNDEZ, A. (2006) “Metodologías activas para la formación en competencias” en Educatio Siglo XXI, vol. 24, p. 35-56.

ICE - INSTITUTO DE CIENCIAS DE LA EDUCACIÓN. UNIVERSITAT POLITÈCNICA DE VALĖNCIA (2015) Proyecto Competencias Transversales UPV $<$ http ://www.upv.es/entidades/ICE/info/U0724624.pdf> [Consulta: 27 de marzo de 2017].

LOBATO, C. (2006) “Estudio y trabajo autónomo del estudiante.” En De Miguel, M. Metodología de enseñanza y aprendizaje para el desarrollo de competencias. Madrid: Alianza.

MATARREDONA DESANTES, N. (2017) “La evaluación por pares en el ámbito no presencial.” En Sein Echaluce Lacleta, M.L., Fidalgo Blanco, A. y García Peñalvo, F. J., La innovación docente como misión del profesorado. Actas del IV Congreso Internacional sobre aprendizaje, innovación, y competitividad. CINAIC 2017. Zaragoza: Servicio de Publicaciones Universidad de Zaragoza.

OLTRA-BADENES, R. y GIL-GÓMEZ, H. (2015) “Técnicas de aprendizaje cooperativo: aplicación de metodologías activas en la asignatura de Recursos Humanos en Empresas Industriales”. En: 3rd International Conference on Innovation, Documentation and Teaching Technologies (INNODOCT/15). Valencia: Open Innovation and Coolhunting in Education. 374-382.

PECHAKUCHA (2018) Metodología Pechakucha < http://www.pechakucha.org/faq> [Consulta: 14 de marzo de 2018].

RUIZ, C. (2006) Metodologías docentes ante el EEES.

$<$ http://www.upct.es/contenido/conv_euro/ficheros/54Ponencia\%20Cecilia\%20Ruiz.pdf> [Consulta: 9 de enero de 2018].

SAYÓS, R. (2013) "Las competencias transversales en las titulaciones de grado de la Universidad de Barcelona. Orientaciones para su desarrollo" en Cuadernos de Docencia Universitaria, vol. 27.

SILVESTRE-CERDÁ, J.A., BAS, M.C., LERMA-GARCÍA, M.J., MATARREDONA-DESANTES, N., OLTRA-BADENES, R. y PÉREZ-ESTEVE, E. (2017) PIMECOE - Efective Oral Communication < $<$ htps://pimecoe.wordpress.com/> [Consulta: 11 de marzo de 2018].

VILLA, A. y POBLETE, M. (2007) Aprendizaje Basado en Competencias. Una propuesta para la evaluación de las competencias genéricas. Bilbao: Universidad de Deusto.

UNIVERSITAT POLITÈCNICA DE VALÈNCIA (2012) Competencias transversales. Blog $<$ http://www.upv.es/contenidos/COMPTRAN/info/956832normalc.html> [Consulta: 23 de abril de 2017]. 